\title{
基因组时代生态-遗传学科交叉的现状与前景
}

\author{
钱 韦 \\ (中国科学院微生物研究所, 中国科学院植物生物技术重点实验室, 北京 100080)
}

\begin{abstract}
摘 要 生态学与遗传学的学科交叉首先建立在种群概念基础上, 文献统计表明, 目前这方面的研究以揭示种群 遗传结构的形成和维持机制为主要方向，同时开始注重关键生化途径与环境适应之间的关系。基因组学的快速发 展, 为系统研究生理代谢在生态适应过程中的作用提供了可能性。分离具有重要生态学意义的基因,并对比近缘 类群中基因表达模式的差异成为生态-遗传学科交叉的新生长点。在对研究实例进行分析后提出了此类研究的特 点, 同时从研究思路、分析方法和研究侧重点方面讨论了生态学在新学科交叉领域中所具有的重要价值。

关键词 生态学 基因组学 学科交叉
\end{abstract}

\section{INTERACTIONS BETWEEN ECOLOGY AND GENETICS IN THE GENOMIC ERA}

\author{
QIAN Wei \\ (Laboratory of Plant Biotechnology, Institute of Microbiology, the Chinese Academy of Sciences , Beijing 100080 , China)
}

\begin{abstract}
Ecology and genetics initially overlapped in the concept of population. Preliminary literature demography showed that studies on the formation and maintenance of population genetic structure are becoming mainstream approaches at the present time. With the rapid development of genomics, cloning ecologically important genes and comparing their subtle expression patterns has become a new field of study. By analyzing characteristics of related cases, it is proposed that ecology will provide important contributions towards the solution of questions arising from gene-oriented studies.
\end{abstract}

Key words Ecology, Genetics , Interaction

1990 年前后, 随着大规模植被调查与研究的完 成, 国内植物生态学界开始寻找新的研究生长点, 直 接导致了种群生物学和分子生态学等生态-遗传交 叉学科在国内的起步。时隔 10 年, 随着拟南芥 (Arabidopsis thaliana) 基因组的基本测定和水稻基因 组框架的提出, 再次激起了有关生态学与分子遗传 学相互借鉴的讨论。对于学科交叉的必要性, 此前 已有学者阐明(张大明，2001; 张大勇，2001) , 本文 不再赘述。目前, 学科交叉已经不是 有必要”, 而是 “如何做” 甚至 如何追” 的问题。为此, 结合近期的 研究, 本文对生态遗传学科交叉的情况进行了讨 论, 并提出与近期再次出现的, 即 生态学是一门软 科学” (Smith，2000)不同的观点。

由于生态学与遗传学在思想方法上有较大差 异, 学科交叉不可能是学科替代, 而是相互借鉴的问 题。尽管遗传学 (或基因组学)是当前生命科学中的 主流学科, 但具体到学科交叉问题上, 遗传学的影响 更多地局限于种群以下层次。在群落以上层次, 遗
传关系一般不是生态系统中的主导生物学关系, 亲 缘问题比起这些生物层次所涉及的生态问题来也显 得较为次要。据此, 本文所讨论的生态-遗传学科交 叉将限制在种群生态学和生理生态学范围内。而从 学科发展的历史看, 生态-遗传两门学科的相互影响 也确实是从种群层次开始的, 时间则可上溯至 20 世 纪 20 年代。

\section{1 以 种群” 概念为基础的交叉研究}

生态学中的 种群” 概念泛指生活在同一时期、 同一地点中同种生物的集合 (Harper , 1977) ,而遗传 学中的 种群” 概念特指共享同一基因库的孟德尔种 群(Merrell，1981)。概念内涵虽略有差异, 但并未妨 碍两门学科抛弃 模式” (Type)物种概念对遗传多样 性的先验否定, 继而在种群概念基础上形成交叉。 Turresson(1922) 和 Clausen 等 (1947) 在 20 世纪 20 年 代开创的实验种群生物学探索, 揭示了种群内存在 着的遗传异质性, 阐明了种群分化与物种形成之间 
的关系, 最终促成了进化论与遗传学的现代综合 (Dobzhansky，1937)。该理论将生态学与遗传学统 一到一个逻辑体系中, 认为物种进化就是种群内的 基因频率在进化动力学因素 (突变、选择、遗传漂变 和基因流动)的联合推动下发生改变, 进而影响种群 适合度的过程 (Hartl \& Clark, 1997)。在此过程中， 生态学因子通过影响各种动力因素, 促使种群遗传 结构发生变化, 最终对物种的进化做出贡献。由此, 生态-遗传学科交叉从一开始就把注意力集中在如 何揭示种群的遗传结构，以及遗传结构如何形成与 维持这两个基本问题上。种群生物学及分子生态学 都以系统解决上述基本问题作为其主要的研究内 容。

学科交叉的进展可以从期刊 Molecular Ecology 来分析。自创刊以来, 该期刊的影响因子就与主流 生态学期刊相近, 且集中刊载生态-遗传学科交叉方 面的论文,因此, Molecular Ecology 的学术水平反映 了学科交叉方面基本的进展情况。为此, 作者根据 该期刊上论文的摘要和关键词, 统计了近 5 年来 （1997～2001）该期刊的原始论文 (Original articles)、 短讯 (Short communications) 中所有植物分子生态学 方面文章的数量, 并根据其研究内容分为几个类别 进行分析(图 1)。分析表明 ,1) Molecular Ecology 中 的论文以研究动物为主, 植物研究均以种群概念为 基础，占文章总数的 15\% 20\% , 最多 24.5\%(1999 年)。植物分子生态学无论是在研究数量上还是在 研究广度上均落后于动物分子生态学。2) 从研究方 向来说, 以植物为对象的论文偏重于探索种群遗传 结构形成和维持过程中的具体机制。虽然直接揭示 种群遗传结构式样的论文数量在各年中都占优势， 但主要是极珍稀濒危物种的研究, 或是具有较好的 生态学研究作为支撑。值得注意的是从叶绿体 DNA 出发常可进行种子流、网状进化等方面的深入 研究, 其数量较多, 因而单独列出进行比较。3)该期 刊上发表的其它论文类别, 按数量排列为: 种群历 史、物种形成对遗传结构影响的研究 $>$ 无性系多样 性的研究 $>$ 繁育系统的研究 $>$ 人为干扰对遗传结构 影响的研究。4) 从 2001 年起该期刊开始以综述方 式,引导生态-基因组学的综合研究, 开辟了环境基 因组学” (Environmental genomics) 专栏, 但到 2001 年 为止反映这方面内容的论文数确实极少。另外, Molecular Ecology 上从未发表过植物基因表达模式 与生态适应之间关系的论文。综上所述, 以种群遗 传结构为核心的研究仍是植物分子生态学研究的主
流领域，随着多种分子标记技术和基于 DNA 序列数 据的分析理论的引入, 促进了研究向解析遗传结构 的形成与维持机制方向发展。

然而值得注意的是,由于种群遗传学理论最终 建立在种群适合度与基因频率这两个关键概念上， 前者在野外研究中很难进行精确的分析, 后者则是 一种数量格局性质的标度, 在解释如何影响种群适 合度时常显得缺乏说服力。由此而产生的多样性与 稳定性、多样性与适合性之间的争论至今未有定论。 这使得种群遗传学理论在 Haldane, Fisher 和 Wright 等发展成熟后出现相对保守的倾向 (Hedrick, 1999)。Kimura 在 20 年前曾提出要为精确定量突变 率、选择系数、有效种群大小和迁移率这 4 个核心参 数做出一次努力的愿望 (Kimura，1983) ,但因问题复 杂而从未实现过。因此,近年来的研究多集中在濒 危物种的保护遗传学领域, 而在理论框架方面未出 现关键性的突破。

实际上, 研究者已经注意到上述情况, 目前的种 群生物学研究已开始在理解种群遗传结构的基础 上, 注重研究与种群适合度有密切关系的基因或基 因家族 (Hedrick, 2001)。从研究思路而言, 这与近 年植物功能基因组学提出的研究方向之一正好一 致, 共同形成一个新的生态-遗传交叉研究领域。

\section{2 具有重要生态学意义的基因的交叉研究}

生理代谢的差异是造成不同植物适应不同生态 环境的内在的、决定性的因素。由于基因频率和种 群遗传结构是数量格局方面的参数, 它们不可能反 映代谢方面的精细差别。为此在生态学历史中, 曾 有一段时期通过比较酶活性变化, 光合作用效率等 生理学指标, 探索植物代谢与生态环境之间的相互 关系。由于生理过程受复杂条件的影响，这方面的 研究当时未在方法上获得大的突破(McIntosh, 1985)。近年来随着突变体和基因组分析的飞速发 展, 分离生物基因与以往相比显得略容易, 直接使研 究者再次考虑代谢过程在生态适应中的重要性, 但 将研究的第一步集中在具有重要生态学意义的基因 上(Hedrick，2001)。这一生态-遗传学科交叉的新领 域正在或已经做出突破性的研究成果, 以下略举数 例，借以分析该领域的特征。

\section{1 植物抗病基因 ( $R$ 基因)进化的研究}

植物-病原微生物相互作用是生态学中研究物 种关系的经典问题。自从玉米抗圆斑病基因 $\mathrm{Hml}$ 被克隆以来( Johal \& Briggs，1992) 到目前约有 30 多 


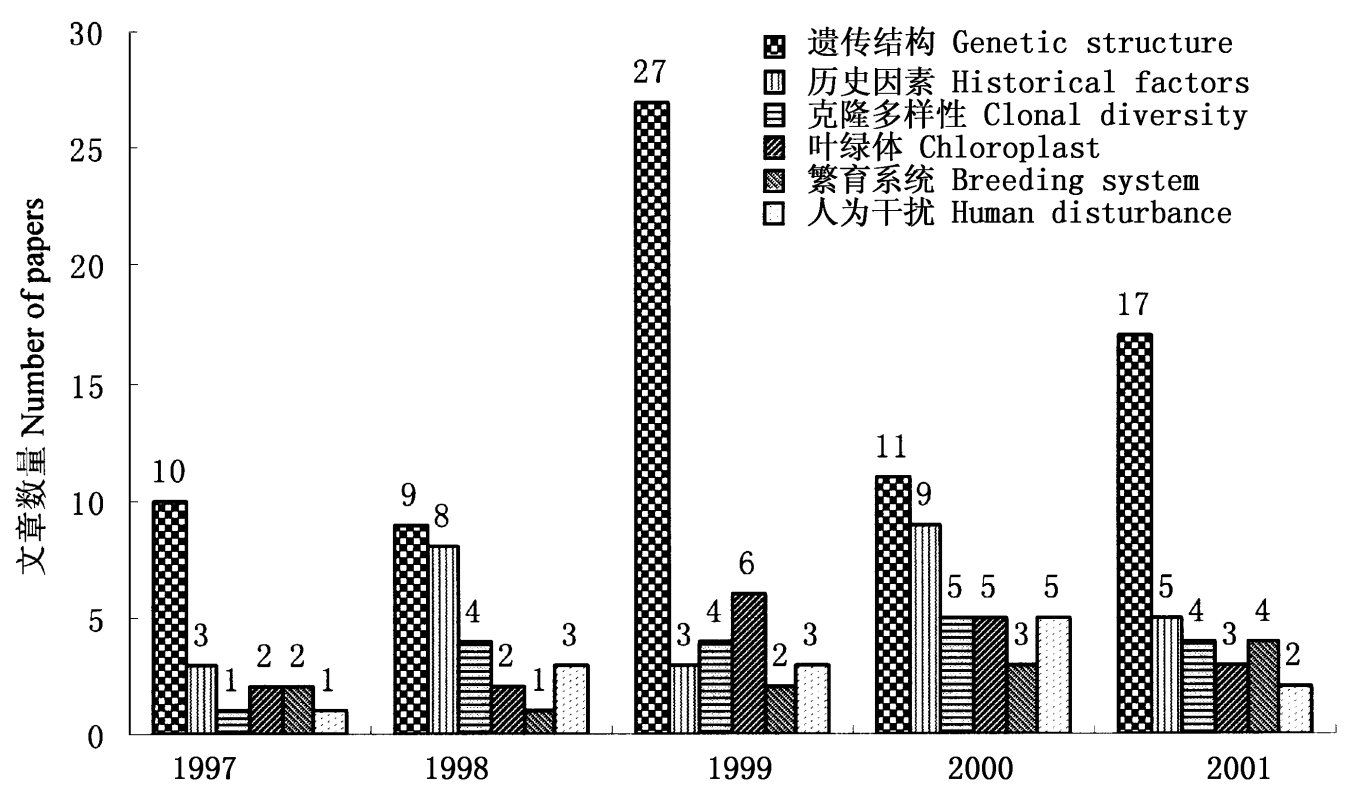

年 Year

图 1 Molecular Ecology 与植物有关论文的数量统计

Fig. 1 Literature demography of plant related papers published on Molecular Ecology

遗传结构: 主要揭示遗传多样性、遗传结构和地理变异式样的论文 历史因素: 物种形成、杂交、自然环境历史变迁对遗传结构影响的论 文 克隆多样性: 以无性系结构为主的论文 叶绿体: 以叶绿体 DNA 为研究方法的论文 繁育系统 研究繁育系统对遗传结构影响的论文 人为干扰 : 转基因生物、生境破坏等对遗传结构影响的论文 Genetic structure : papers for analysis of genetic diversity, structure and geographical variation

Historical factors : papers for revealing of genetic structure with speciation, hybridization and environment Clonal diversity : papers on clonal growth ; Chloroplast : papers that use chloroplast DNA as study method Breeding system : papers on genetic structure affected by breeding systems Human disturbance : papers on the effect by transgenic organism, environmental deterioration.

个植物 $R$ 基因被成功分离。除 $H m l$ 以外的 $R$ 基因 均编码信号转导蛋白，与病原菌的无毒基因 ( avr 基 因)之间存在 基因对基因” (Gene for gene)的遗传关 系。对 $R$ 基因中最大一类，即 NBS-LRR 类 ( Nucleotide binding site, leucine-rich repeats) 基因在植物 生态型或近缘类群中进行的分析表明 (Dangl \& Jones，2001）,由于基因重复、重组、转座和基因转变 (Conversion)的作用, $R$ 具有极高的遗传多态性, 有 利于适应病原微生物的快速进化，发展出新的抗病 专一性 (Caicedo et al . , 1999)。 $R$ 基因的信号感受 区域具有氨基酸非同义替换率 $>$ 同义替换率的特 征极有可能是发生了适应选择 (Adaptive selection) 的结果。它们多以基因家族形式在染色体上成簇存 在 其进化过程中成功地避免了家族一致进化 (Concerted evolution)造成的同源化(即多态性下降)趋势， 其机理尚未完全清楚 (Parniske et al ，1997)。对 $R$ 基因的探索使研究者得以站在信号转导的水平上, 重新认识植物-病原微生物这两种不同遗传系统之 间的协同进化关系。

\section{2 细菌群体感应 (Quroum-sensing) 基因的研究}

群体感应是指细菌能够 感知” 同种个体密度变
化，继而诱导出现特定生理状态，如致病性、生物膜 (Biofilm) 形成的现象。群体感应是微生物生态学研 究中的主题之一 (Miller \& Bassler , 2001)。近年的研 究表明, 群体感应实际受到细菌基因组中相关基因 的精细调控。例如, 当生物发光细菌哈氏弧菌( Vibrio harveyi) 的个体密度达到一定程度时, 发光基因 lux 的表达量提高约 1000 倍,使细菌细胞发出菼 光。这一群体感应现象受到基因 $\operatorname{Lux} N 、 \operatorname{Lux} Q$ 和 LuxO 的调节。它们属于细菌中的双组分信号转导 系统 (Two-component signal transduction system) ,LuxN 为一类组氨酸激酶 (Histidine kinase), 其信号输入区 能监测细菌自身分泌物浓度的变化, 并能通过激酶 区组氨酸的磷酸化, 将磷酸基团传递给下游的效应 调节蛋白 LuxO (Response regulator) ,再由后者的信号 输出区激活相应基因的转录，解除对发光基因 lux 的抑制( Lilley \& Bassler，2000)。到目前为止，已有 许多类似的双组分系统基因被鉴定，它们广泛参与 细菌与外界生态因子之间相互作用的信号转导过 程，包括趋化性、渗透性、狍子形成、致病性等等。对 这些基因的研究证明,在生态因子变化和生物的生 理代谢改变之间, 存在着精细的信号接收与传递机 
制，这些机制的差异对生物的生态分布、适应性和进 化具有重要的影响。

\section{3 控制植物开花时间基因的研究}

在生物学 种” 的概念中, 植物开花时间的差异 是一种重要的生殖隔离机制, 也是生态学中区分某 些植物生态型的标准, 它在植物的进化过程中占有 重要地位 (Stebbins , 1950)。Johanson 等 (2000) 利用 图位克隆法在拟南芥中分离到一个决定花期早晚的 基因 FRI。该基因在斯德哥尔摩生态型中表现为显 性, 其正常表达决定植株为晚花类型。对比 FRI 基 因在 38 种拟南芥生态型中的序列和表达差异发现， 这些生态型大致可分为 5 类。与斯德哥尔摩型相 比, 其中两类生态型在 FRI 位点的不同位置上有缺 失, 使得 FRI 基因的转录被提前中止,相应生态型 均表现为早花类型。第四类在 FRI 位点上有一个 改变氨基酸种类的突变, 但它仍为晚花类型, 表明该 突变不影响 FRI 的正常功能。最后一类虽表现为 早花类型,但研究表明其 $F R I$ 基因的表达与斯德哥 尔摩生态型相同, 所以这一类的早花现象可能不是 由 $F R I$ 基因突变造成的。根据 FRI 基因位点的突 变模式, 研究者还提出了上述 5 种生态型的进化次 序, 而 FRI 基因的变异情况至少与其中 4 种的花期 变化有直接的关系。对 FRI 基因的研究成功地将 生态型分化与基因组成和表达模式的精细差异联系 起来。

类似的研究还包括植物基因组进化的比较分 析、基因与基因组重复的生态学效应、植物光周期基 因遗传分化与环境变迁的关系、植物多倍体中基因 沉默的机理、植物表型可塑性的生态学和分子遗传 学基础 数量性状位点 (QTL) 与表型适应性的关系 等一系列热点问题 (Mitchell-Olds, 2001)。正如 Somerville 和 Dangl (2000) 指出的那样, 此类研究也 是未来十年内植物基因组学研究的重要方向, 其问 题的本质属于生态学, 但因问题的特点, 可以还原到 基因调控的水平, 来探索植物对异质环境的适应性。 从目前来看此类研究具有如下特征:1) 研究以揭示 生理代谢与生态适应的关系为目的, 目前集中于分 离具有重要生态学意义的基因, 并对比其基本表达 模式 2)建立以模式物种为对象的研究体系, 特别是 十字花科植物 (以拟南芥为核心) 、禾本科植物 (以栽 培稻为核心)和其它一些基因组学研究基础较好的 植物类群 3) 开始运用功能基因组学的实验手段, 特 别是微阵列分析 (Microarray)、大规模比较基因组作 图 (Large-scale genome mapping) 等高通量分析方法来
解析生态现象的复杂性 (Gibson，2002)。这方面的 研究无疑将是未来十年生态-遗传学科交叉研究的 一个新领域。

当然,基因组学渗入的同时也产生了学科之间 相互竞争的问题，如果这些经典生态学问题都已经 成功地还原到了基因水平, 那么生态学是否处于一 种仅仅提出问题, 却必须交由基因组学研究去解决 的尴加境地?

\section{3 在基因组时代生态学研究什么?}

可以预见的是, 新领域的出现必将引发种群和 生理生态学层次的重大突破, 人类将在理解生物的 环境适应性方面进入到生态因子与基因调控相互作 用的水平。然而, 这种重大突破并不意味着种群和 个体生态学研究会被纯粹的基因组学所替代。如果 是这样, 则基因组学的渗入即降格为仅是为了填补 生态学的遗传或生化空白, 而对于生态学而言, 则也 许是在细节得以完整的情况下，学术思想的一次倒 退。这样的学科交叉完全背离了原有的意义。在有 效的学科交叉中, 生态学不但要提出问题, 而且也将 在解决问题中发挥关键性的作用。

生态学是研究生物之间和生物及其环境之间相 互关系的一门科学 和进化论一样, 系统思维是其学 术思想的核心。生态学有异于实验生命科学的一个 特点, 即在于它强调生命现象中的过程而非实体，以 及过程之间发生的相互作用(Odum, 1971)。在此思 想主导下, 从英美学派开始, 生态学就发展了一整套 定量的分析过程及其相互作用的模型化方法。模型 分析在种群生物学领域更是达到了相当的程度 (Hartl \& Clark, 1997)。相比之下, 分子遗传学(或基 因组学) 是在统一了历史上的结构学派、信息学派和 生化遗传学派发展形成的, 尽管它也重视过程与相 互作用(如基因之间的相互作用)，但在此前一直都 不是其研究的主攻方向, 目前还缺乏有力的研究工 具。20 世纪 50 60 年代分子遗传的学术思想甚至 有向机械还原论倒退的趋势 (Allen , 1975)。因此, 在涉及生态-遗传学科交叉的新问题中，只有引入生 态学的系统思想与强大的数理分析能力, 才有可能 回答仅仅依靠当中任一学科而无法穷尽的问题。以 下结合前述实例加以具体分析。

3.1 在问题涉及进化历史和生态关系变迁时, 仅从 分子遗传学出发易做出片面理解

前述植物 $R$ 基因遗传多样性的研究中存在两 种可能的进化模式,一种被称为 军备竞赛” (Arm- 
race) 即植物 $R$ 基因和病原微生物的无毒基因 ( avr 基因)之间存在协同进化关系，双方各针对对方的变 化而做出持续不断的调整，由此而造成了 $R$ 基因极 度的遗传多样性。另一种模式被称之为 堑壕战” （Trench war）意指 $R$ 基因与 $a v r$ 基因之间的关系由 来以久 其遗传多样性的历史可上溯到两种基因的 产生初期 此后则依靠频率制约选择来维持遗传多 态(Bergelson et al . ,2001)。目前研究这两种进化模 式的思路是对比 $R$ 基因在近缘物种中分化的时间： 如果 $R$ 基因分化甚至早于物种分化，则 军备竞赛” 理论很难获得支持(Riely \& Montin ,2001)。显然，这 个根本性问题的解决不可能由基因表达模式来推 断, 因为它涉及到物种分化的时间。如果从生态学 方面进行思考，则上述两个进化模式假设的有效性 涉及同一生态学前提，即它们都要求 $R$ 基因与 $a v r$ 基因相互作用的模式在当前和在进化历史中相同 (即古今一致)。如果这种相互关系不符合古今一致 的前提，则就需要考虑新的进化模式或进化模式在 不同进化阶段中的转变。因此, 当问题涉及进化历 史和生态关系时，仅从分子遗传角度思考可能提出 无效问题或做出片面理解。

3.2 在问题涉及过程之间的相互作用时, 需要利用 生态学发展成熟的分析方法

与实验条件相比, 生物在自然生境中要适应复 杂得多的生态因子的影响。这使得生物通过长期进 化形成的相应的信号转导机制来感受外界环境的刺 激, 以此对自身行为和生理过程作出调整。这些信 号系统之间普遍存在着以信号对答 (Crosstalk) 为特 点的相互作用方式 (Schaechter et al. ,2001)。以细 菌为例, 当其受到环境胁迫时, 就必须对细胞的营养 代谢、鞭毛摆动方式、胞外蛋白分泌等过程进行系统 化的调节。实验室条件毕竟比自然条件简化，因此， 如何分析复杂条件下细胞信号转导中的网络特征是 目前研究的一个难题。除了继续对其进行分子遗传 学分析外, 利用数学模型将有可能为理解信号网络 的作用机理提供帮助。Neidhardt 和 Savageum (1996) 即发展了一种称为 Power-law”的数学模型, 分析在 不同的生境中 细菌基因表达模式变化的细胞动力 学特征。比如该模型分析表明,当环境刺激要求基 因具有高水平的转录时, 细菌细胞一般表现出正向 调控的动力学特征, 当要求基因的转录水平下降时, 则表现为负向调控的动力学特征。这一模式在多个 代谢过程中获得实验的支持, 并正在探索该调控模 式的分子机理。显然, 当生态适应涉及代谢途径的
功能冗余 (Redundancy)、信号网络 (Network)、系统备 份 (Backup)、混沌行为 (Chaos) 等与过程间相互作用 有关的特征时, 在类似系统化过程中作出贡献的生 态学将为研究提供更有力的分析手段。

3.3 自然进化的过程决定了生态学和遗传学侧重 于回答问题的不同方面

Stebbins (1999)将物种的进化总结为两个阶段： 首先是种群以某种方式产生遗传变异, 其次是在不 同生态因子影响下, 变异在种群内固定或消失。第 一个过程主要与基因组的突变性质有关, 是分子遗 传学研究的重点。第二个过程则必须从生态学或进 化生物学角度来探讨。就上述调控开花时间的 FRI 基因来说, 虽然不同突变模式是造成不同生态型花 期分化的主要遗传学原因，但从生态分布上来看，生 态型之间的遗传分化与地理分布却关系不大。研究 者推测其进化的动力来源于人类造成的选择压力 (Johanson et al. ，2000)，可是具体的选择压力是什 么? 什么原因使上述生态型在可以进行基因交流的 情况下却发生了遗传分化? 早花突变体产生后又是 哪种因素使之在种群中扩散, 最终固定在某一生态 分布区内 ? 如果早花突变体获得了一定的选择有利 性 那么在它固定的过程中 种群以何种方式适应随 之而来的遗传负荷? 显然, FRI 基因的成功克隆在 回答了早晚花生态型的遗传基础时, 仍未解决经典 的生态学问题, 其贡献在于它使这些生态学问题的 研究获得一个新起点。

由此可见，复杂多样的生态适应现象不可能总 由同一种方法加以探索, 而基因组学近年的飞速发 展, 为更深层次的学科交叉提供了基础。显然, 这样 的学科交叉要求分子遗传学家从生态学角度系统地 思考问题，并理解模型分析的实质; 而对于野外生态 学家来说, 则要应用与样地调查完全不同的基因组 学工具与分析思路。双方都懂得对方的语言, 必然 能进行卓有成效的合作，达到系统理解生态适应的 目的。

\section{4 结 论}

从长远来看生态遗传的学科交叉是双赢的, 不过就目前而言, 学科交叉确实表现为生态学对遗 传学思想方法的借鉴与应用, 前面已经论述了这只 代表学科交叉的一个过程。但如果即由此认为实验 背景较弱的生态学是一门软” 科学, 前面已述及这 有悖于两门学科交叉的目的。从事野外生态学的研 究者常常要面对不易简化的生态学难题, 其中许多 
都是科学目前的发展水平或依靠科学本身无力加以 解决的。这也是生态学被视为 软” 科学的又一原 因。然而无法否认的是, 近 40 年来, 正是生态学的 发展对人类社会的生存产生了最重要的影响: 社会 可持续发展理论的建立、自然保护运动的兴起和全 球环境污染的监控等等一系列事关人类生存的重大 问题, 无一不出自于生态学, 并在其领域内寻找系统 的解决方案。杰出的野外生态学家及分子病理学 家,普利策新闻文学奖及生态学泰勒奖获得者 Jared Diamond 在被问及生态学研究有无意义时, 恰如其 分地表达了他对生态学的评价“: 对全社会而言 现 代科学、生物学和社会中最重要的问题都不是实验 科学所能解决的那种问题。真正威胁我们地球是否 有居住价值以及从现在开始 50 年内人类生存的, 是 对我们所处生物环境的破坏。因此我认为, 野外科 学、生态学和进化学是对人类社会维持最为重要的 生物学领域(Wolpert \& Richards, 1997)。”这句话将 有益于我们在进行生态-遗传学交叉研究时正确思 考生态学所处的地位。

\section{参 考 文 献}

Allen, G. E. 1975. Life science in the twentieth century. London: Cambridge University Press. $254 \sim 259$.

Bergelson, J., M. Kreitman, E. A. Stahl \& D. Tan. 2001. Evolutionary dynamics of plant $R$-genes. Science, 292: $2281 \sim$ 2285 .

Caicedo, A. C., B. A. Schaal \& B. N. Kunkel. 1999. Diversity and molecular evolution of the RPS2 resistance gene in Arabidopsis thaliana. Proceedings of the National Academy of Sciences of the United States of America, 96: $302 \sim 306$.

Clausen, J., D. D. Keck \& W. M. Hiesey. 1947. Heredity of geographically and ecologically isolated races. American Naturalist, 81: $114 \sim 133$.

Dangl, J. L. \& J. D. G. Jones. 2001. Plant pathogens and integrated defence responses to infection. Nature, 411:826 833.

Dobzhansky, Th. 1937. Genetics and the origin of species. New York: Columbia University Press. 3 18.

Gibson, G. 2002. Microarrays in ecology and evolution: a preview. Molecular Ecology, 11: $17 \sim 24$.

Harper, J. L. 1977. Population biology of plants. London and New York: Academy Press. $1 \sim 23$.

Hartl, D. L. \& A. G. Clark. 1997. Principles of population genetics. Massachusetts: Sinauer. $37 \sim 73$.

Hedrick, P. W. 1999. Perspective: high variable loci and their interpretation in evolution and conservation. Evolution, 53:313 318 .

Hedrick, P. W. 2001. Conservation genetics: where are we now? Trends in Ecology and Evolution, 16: 629 636.

Johal, G. S.\& S. P. Briggs. 1992. Reductase activity encoded by the $H m l$ disease resistance gene in maize. Science, 258:985 987.
Johanson, U., J. West, C. Lister, M. Scott, R. Amasino \& C. Dean. 2000. Molecular analysis of FRIGIDA, a major determinant of natural variation in Arabidopsis flowering time. Science, 290: $347 \sim 349$.

Kimura, M. 1983. The neutral theory of molecular evolution. London: Cambridge University Press. 319 409.

Lilley, B. N.\& B. L. Bassler. 2000. Regulation of quorum sensing in Vibrio harveyi by LuxO and sigma-54. Molecular Microbiology, 36: $688 \sim 696$.

McIntosh, R. P. 1985. The background of ecology: theories and concept. London: Cambridge University Press.

Merrell, D. J. 1981. Ecological genetics. London: Longman. 1 4.

Miller, M. B. \& B. L. Bassler. 2001. Quorum sensing in bacteria. Annual Review of Microbiology, 55:165 199.

Mitchell-Olds, T. 2001. Arabidopsis thaliana and its wild relatives: a model system for ecology and evolution. Trends in Ecology and Evolution, 16:693 700.

Neidhardt, F. C. \& M. A. Savageau. 1996. Regulation beyond the operon. In: Neidhardt, F. C., R. Curtiss, J. L. Ingraham, E. C. C. Lin, K. B. Low, B. Magasanik, W. S. Reznikoff, M. Riley, M. Schaechter \& H. E. Umbarger eds. Escherichia coli and Salmonella: cellular and molecular biology. Washington: ASM Press. 1310 1324 .

Odum, E. P. 1971. Fundermentals of ecology. Philadelphia: Saunders. $3 \sim 12$.

Parniske, M., K. E. Hammond-Kosack, C. Golstein, C. M. Thomas, D. A. Jones, K. Harrison, B. B. Wulff \& J. D. Jones. 1997. Novel disease resistance specificities result from sequence exchange between tandemly repeated genes at the $C f-4 / 9$ locus of tomato. Cell, 91: $821 \sim 832$.

Riely, B. K. \& G. B. Martin. 2001. Ancient origin of pathogen recognition specificity conferred by the tomato disease resistance gene Pto . Proceedings of Natural Academy of Sciences of the Unite States of America, 98:2059 2064.

Schaechter, M. \& The View from Here Group. 2001. Escherichia coli and Salmonella 2000: the view from here. Microbiology and Molecular Biology Reviews, 65:119 130 .

Smith, J. 2000. Nice work - but is it science? Nature, 408:293.

Somerville, C. \& J. Dangl. 2000. Genomics: plant biology in 2010. Science, 290:2077 2078 .

Stebbins, G. L. 1950. Variation and evolution in plants. New York: Columbia University Press. $42 \sim 71$.

Stebbins, G. L. 1999. A brief summary of my idea on evolution. American Journal of Botany, 86:1207 1208.

Turresson, G. 1922. The genotypical response of the plant species to the habitat. Hereditas, 3:211 350.

West, A. H. \& A. M. Stock. 2001. Histidine kinase and response regulator proteins in two-component signaling systems. Trends in Genetics, 26:369 376 .

Wolpert, L. \& A. Richards. (transtated by Ke, X. R. (柯欣 瑞) ). 2000. Passionate minds: the inner worlds of scientists. Shanghai: Shanghai Scientific and Technological Education Publishing House. 46.

Zhang, D. M. (张大明). 2001. Ecology and genetics. Acta Phytoecologica Sinica (植物生态学报), 25:252 253. (in Chinese)

Zhang, D. Y. (张大勇). 2001. Ecology, genetics, and Evolution. Acta Phytoecologica Sinica (植物生态学报), 25:254. (in Chinese) 